\title{
Perilunate Injuries as an Important Cause of Hand Morbidity
}

\author{
(D) Bülent Karslıoğlu, (D) Ersin Taşatan, (D) Süleyman Semih Dedeoğlu, (D) Hakan Gürbüz \\ University of Health Sciences Turkey, Prof. Dr. Cemil Taşcıoğlu City Hospital, Clinic of Orthopedics and Traumatology, Istanbul, Turkey
}

\section{Abstract}

Objective: Perilunate injuries are rare but highly overlooked injuries among hand injuries. These injuries, primarily seen in the young population after high-energy trauma, cause significant disability or loss of labor. We aimed to share the 2-year follow-up results of patients who were operated on for perilunate injury.

Methods: Twelve patients diagnosed with perilunate injuries were included in the study. Seven patients had trans-scaphoid, three patients had trans-scaphoid and trans-radial, and two patients had trans-scaphoid and transcapitate fractures. Five cases were operated on using the dorsal approach, three cases with the volar, and the remaining four cases using the combined approach.

Results: The patients were followed for an average of 25.7 (range, 17-34) months. At the end of the follow-up period, scaphoid avascular necrosis was observed in two patients and lunate avascular necrosis in one patient. Three patients were in stage 4 wrist osteoarthritis; two patients were in stage 3; six patients were in stage 2, and one patient was in stage 1. Four cases were evaluated as good, five cases were fair, and three were poor according to the modified Green and O'Brien clinical evaluation scale.

Conclusion: Perilunate injuries are rare and the most important step in making the suspected diagnosis. Early treatment prevents carpal arthrosis and loss of wrist motion. Care should be taken during diagnosis and treatment. Also, patients should be informed at every stage because perilunate injuries cause permanent morbidity in young patients.

Keywords: Perilunate, fracture, ligament dissociation

\section{INTRODUCTION}

High-energy injuries to the wrist are most common in patients under 40 with high functional demand expectations. Depending on the position of the wrist at the time of trauma and the direction of the trauma forces, one or more conditions may occur: distal radius fracture, radiocarpal or perilunate dislocations (PLDS), or other intercarpal dislocations (scaphoid, capitate, trapezium, or hamate) (1). Among these conditions, PLDs are relatively less common and often overlooked during the first examination. PLDs constitute $7 \%$ of all carpal bone traumas (2). PLDs and perilunate fracture dislocations (PLD-PLFDs) are often difficult to diagnose. The main problem with carpal ligament injuries is that they cause arthrosis in the medium or long term (starting from the radiocarpal joint and extending to the intercarpal joints (3). Early treatment of perilunate injuries to restore wrist motion and function prevents complications, such as chronic carpal instability and posttraumatic arthrosis, because reconstructive procedures are limited. Our study aims to share the 2-year follow-up results of patients operated on for perilunate injury.

\section{METHODS}

Patients diagnosed with perilunate injuries were among the patients admitted to the emergency department because of hand trauma between January 2015, and January 2018 were included in this retrospective study. Fifteen patients were selected for inclusion in the study because of a diagnosis of perilunate injury. Three patients did not participate in the study because they were out of follow-up visits. Twelve patients were included in the study. This article complies with the Ethical Principles for Medical Research Involving Human Subjects of the 
World Medical Association Declaration of Helsinki. University Health Science Turkey, Prof. Dr. Cemil Tașçıŏlu Hospital Ethical Committee (no: 48670771-514.10).

All patients were male. The mean patient age was 32.4 (range, 24-43) years. All cases were due to high-energy trauma occurring after a fall. Falling was due to sports in five patients, whereas the remaining seven patients fell from a certain height due to an accident. The average time from trauma to surgery was 3.4 (range, 1-9) days. Three patients were misdiagnosed at the initial examination but were operated on when they re-applied more than five days after the trauma (six days, eight days, and nine days). The remaining nine patients were operated on as acute cases within the first five days after trauma (Table 1). All patients were evaluated with preoperative and postoperative standard wrist anteroposterior (AP), and lateral (LAT) radiographs, and computed tomography (CT) examinations were performed for patients warranting confirmation.

According to the Herzberg classification, seven patients had trans-scaphoid, three patients had trans-scaphoid and transradial, and two patients had trans-scaphoid and transcapitate (TC) fractures $(4,5)$. Five cases were operated using the dorsal approach, three cases using the volar, and the remaining four cases were operated on using the combined approach. Herbert screws or K-wires were used to treat scaphoid fractures. The scapholunate, capitolunate, and lunotriquetral joints were stabilized with K-wires. Mini anchors were used in patients who underwent ligament repair (Figure 1).

\section{Statistical Analysis}

SPSS (Statistical Package for Social Sciences) 21.0 and Microsoft Office Excel 2016 programs were used for statistical analysis.
Shapiro-Wilk test was used to determine whether the study findings were normally distributed. Comparisons between groups were made using an independent t-test or analysis of variance.

\section{RESULTS}

The patients were followed for an average of 25.7 (range, 17-34) months. At the end of the follow-up period, scaphoid avascular necrosis and then scaphoid non-union advanced collapse in two patients, and lunate avascular necrosis was observed in one patient. According to the osteoarthritis classification, the radiological wrist evaluations indicated that (6) three patients were in stage 4 , two patients were in stage 3 , six patients were in stage 2, and one patient was in stage 1. According to the modified Green and O'Brien clinical evaluation scale, three cases were evaluated as good, five were fair, and three

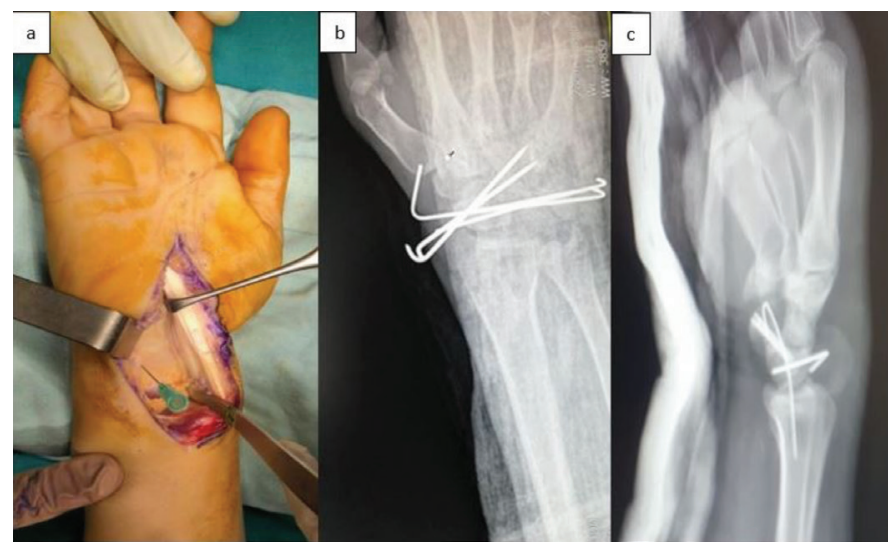

Figure 1. (a) Stage 3 perilunate injury treated with the volar approach and $(b, c)$ postoperative $X$-ray images

\begin{tabular}{|l|l|l|l|l|l|}
\hline \multicolumn{6}{|l|}{ Table 1. Statistical data of the patients included in the study } \\
\hline Patients & Age (years) & Fracture type & Approach & Trauma to surgery (days) & Follow-up time (months) \\
\hline 1 & 43 & T-S & Dorsal + volar & 6 & 25 \\
\hline 2 & 34 & T-S & Volar & 3 & 18 \\
\hline 3 & 25 & T-RS-S & Dorsal + volar & 2 & 32 \\
\hline 4 & 30 & T-S & Dorsal & 2 & 27 \\
\hline 5 & 23 & T-S & Volar & 9 & 25 \\
\hline 6 & 26 & T-RS-S & Volar & 3 & 23 \\
\hline 7 & 42 & T-S & Dorsal & 2 & 34 \\
\hline 8 & 24 & T-S-C & Dorsal + volar & 1 & 33 \\
\hline 9 & 35 & T-RSC-S & Dorsal & 2 & 17 \\
\hline 10 & 34 & T-S & Dorsal & 8 & 27 \\
\hline 11 & 39 & T-S-C & Dorsal + volar & 1 & 17 \\
\hline 12 & 34 & T-S & Dorsal & 2 & 31 \\
\hline T-S: Transschaphoid, T-RS-S: Transscaphoid and transradial, T-S-C: Transscaphoid and transcapitate & \\
\hline
\end{tabular}


were poor. All the poor cases were delayed cases. The clinical findings of two patients who complained of numbness in their fingertips showed that their median nerve compression regressed postoperatively.

\section{DISCUSSION}

PLDs constitute $7 \%$ of all carpal bone traumas (2). Axial load that occurs due to falling on an outstretched hand causes hyperextension and ulnar deviation of the wrist. The resulting intercarpal supination causes progressive perilunar instability. These injuries occur sequentially because of progressive ligamentous disruption. The load starts from the radial side and begins to transmit through the scaphoid or scapholunate ligament interval. This force causes a trans-scaphoid fracture or scapholunate ligament dissociation. The force then spreads toward the midcarpal area and ends at the ulnar part of the wrist (Figure 2) (7).

The severity of the trauma determines the pathways of two clinical situations: Either a PLD or PLFD on the posteroanterior or AP view. Mayfield et al. (8) demonstrated that progressive perilunate injuries occur in four stages because the disorientation of the carpal bones around the lunate creates the PLD. The scapholunate and radioscaphocapitate ligaments are disrupted in stage 1. The lunocapitate ligament disruption occurs in stage

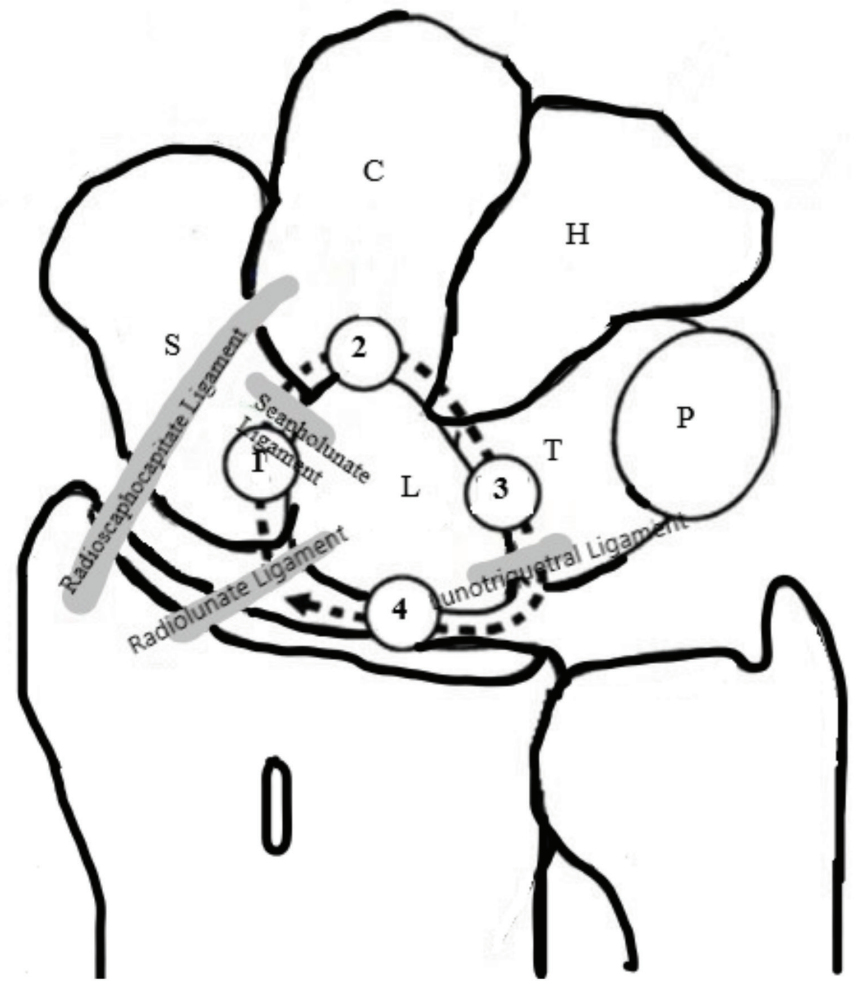

Figure 2. The force applied to the wrist, starting from the radial side, is transmitted through the ligaments toward the ulna
2. The lunotriquetral joint disruption (the entire carpus separates from the lunate) in stage 3 and volar lunate dislocation due to weakness in the volar capsule into the carpal tunnel occurs in stage 4.

Gilula's (9) arcs are formed by the proximal and distal articular surfaces of the proximal row and the proximal cortical margins of the capitate and the hamate. Perilunate instability can be described in the form of the lesser arc and greater arc injuries. Lesser arc injuries occur because of pure and complete ligamentous disruption, which occurs on the second and third Gilula's (9) arc. Greater arc injuries occur at the most proximal arc among the three radiographic arcs described by Gilula (9) in the posteroanterior or AP wrist radiographs. These injuries are ligamentous and osseous. This group includes intact scaphoid [transradial-styloid (TRS), TC, transtriquetrum (TT), and combinations], and the trans-scaphoid, PLFD, and their variants (TS-TRS, TSTC, TS-TT, and combinations). The most commonly seen perilunate instability is the trans-scaphoid PLFD (10). In our study, trans-scaphoid PLFD was the most common and was observed in seven patients.

In the first examination and radiographic evaluation, 25\% of the cases can be missed (5). A study has revealed that the lack of experience is the most important determining factor in the missed diagnosis of perilunate injuries (11). Although there is no obvious deformity, edema, pain, limited movement of the wrist joint, and even symptoms related to acute median nerve compression in $24 \%-45 \%$ of patients can be observed $(12,13)$. Among our patients, three patients were operated on as delayed cases because they were not diagnosed during the first examination. These patients had the worst Green and O'Brien clinical evaluation scores.

Since perilunate injuries are frequently overlooked, the functional results of patients who undergo delayed surgery are poor. Studies have shown that patients undergoing surgery have progressive, permanent damage to the radiocapitate and midcarpal joints even two months after the first injury (14). For this reason, poor postoperative results are thought to be associated with delayed surgery, overlooked initial diagnosis, and concomitant carpal bone injuries (15).

Standard wrist PA and LAT radiographs are usually sufficient for diagnosis. The gapping in the carpal bone joint faces, distortions in the continuation of the Gilula (9) arcs, overlapping of the carpal bones, and loss of carpal height may help make a diagnosis. CT may help to detect occult fractures and fracture type. Occult fractures, bone bruises, and ligamentous disruptions can be seen with magnetic resonance imaging (MRI). CT or MRI, 
if necessary, will prevent cases from being overlooked, especially for suspected cases.

Historically, although the treatment of perilunate injuries has been cast after closed reduction, studies have shown that anatomic reduction of the intercarpal relationship is the key to avoid carpal instability and avascular necrosis (13,16-18). Inadequate reduction of the carpal bones is associated with arthritis, chronic persistent pain, scapholunate advanced collapse, carpal instability, and loss of wrist motion (7). Although it does not restore anatomical carpal alignment, the closed reduction should attempt to reduce the pressure on the median nerve for every patient waiting in the emergency room for definitive treatment. A closed reduction maneuver has been tried in all our acute cases, from diagnosis to surgery. The primary treatment should be surgery because 59\% reduction loss was detected within six weeks in patients who had a plaster cast after closed reduction (19).

The volar approach, dorsal approach, combined approach, or arthroscopic-assisted open surgery may be preferred. The optimal surgical approach is still controversial. The dorsal approach has advantages, such as better reduction of the scapholunate interval, allowing the repair of the dorsal ligaments, and better visualization of the proximal carpal row and midcarpal row. The high probability of developing avascular necrosis of the scaphoid and lunate is its most important disadvantage (20). The volar approach has the advantages of carpal tunnel decompression, better repair of volar ligaments (especially the lunortriquetral ligament) but has disadvantages such as difficult access to distal scaphoid fractures (21). The type of injury is important in addition to the surgeon's experience in determining the surgical approach. In our study, surgery was performed using the combined approach in four patients, the volar approach in three patients, and the dorsal approach in five patients. Especially in patients whose distal scaphoid fractures could not be reached by the volar approach, either the combined approach was preferred or started with the dorsal approach.

\section{CONCLUSION}

Perilunate injuries are rare and the most important step in making the suspected diagnosis. Early treatment prevents carpal arthrosis and loss of wrist motion. The risk of posttraumatic arthritis is high in the long term due to chondral damage that occurs depending on the severity of the trauma despite appropriate treatment. Care should be taken during diagnosis and treatment. Also, patients should be informed at every stage because perilunate injuries cause permanent morbidity in young patients. Such injuries should not be overlooked to avoid medical and legal problems.

\section{Ethics}

Ethics Committee Approval: This article complies with the Ethical Principles for Medical Research Involving Human Subjects of the World Medical Association Declaration of Helsinki. University Health Science Turkey, Prof. Dr. Cemil Taşçıŏlu Hospital Ethical Committee (no: 48670771-514.10).

Informed Consent: An informed consent form was signed by all patients.

Peer-review: Externally peer-reviewed.

\section{Authorship Contributions}

Surgical and Medical Practices: B.K., H.G., Concept: B.K., S.S.D., Design: B.K., Data Collection or Processing: E.T., Analysis or Interpretation: E.T., S.S.D., Literature Search: E.T., Writing: B.K.

Conflict of Interest: No conflict of interest was declared by the authors.

Financial Disclosure: The authors declared that this study received no financial support.

\section{REFERENCES}

1. Obert L, Loisel F, Jardin E, Gasse N, Lepage D. High-energy injuries of the wrist. Orthop Traumatol Surg Res 2016;102(Suppl 1):S81-93.

2. Weil WM, Slade JF 3rd, Trumble TE. Open and arthroscopic treatment of perilunate injuries. Clin Orthop Relat Res 2006;445:120-32.

3. Chantelot C. Post-traumatic carpal instability. Orthop Traumatol Surg Res 2014;100(Suppl 1):S45-53.

4. Herzberg G, Forissier D. Acute dorsal trans-scaphoid perilunate fracture-dislocations: medium-term results. J Hand Surg $\mathrm{Br}$ 2002;27:498-502

5. Herzberg G, Comtet J], Linscheid RL, Amadio PC, Cooney WP, Stalder J. Perilunate dislocations and fracture-dislocations: a multicenter study. J Hand Surg Am 1993;18:768-79.

6. Weiss KE, Rodner CM. Osteoarthritis of the wrist. The Journal of Hand Surgery 2007;32:725-46.

7. Muppavarapu RC, Capo JT. Perilunate dislocations and fracture dislocations. Hand Clinics 2015;31:399-408.

8. Mayfield JK, Johnson RP, Kilcoyne RK. Carpal dislocations: pathomechanics and progressive perilunar instability. J Hand Surg Am $1980 ; 5: 226-41$

9. Gilula LA. Carpal injuries: analytic approach and case exercises. AJR Am J Roentgenol 1979;133:503-17.

10. Garg B, Batra S. Chronic trans-scaphoid perilunate dislocation: Current management protocol. JCOT 2020;4:523-8.

11. Çolak I, Bekler HI, Bulut G, Eceviz E, Gülabi D, Çeçen GS. Lack of experience is a significant factor in the missed diagnosis of perilunate 
fracture dislocation or isolated dislocation. Acta Orthop Traumatol Turc 2018;52:32-6.

12. Hildebrand KA, Ross DC, Patterson SD, Roth JH, MacDermid JC, King GJ. Dorsal perilunate dislocations and fracture-dislocations: questionnaire, clinical, and radiographic evaluation. J Hand Surg Am 2000;25:1069-79.

13. Knoll VD, Allan C, Trumble TE. Trans-scaphoid perilunate fracture dislocations: results of screw fixation of the scaphoid and lunotriquetral repair with a dorsal approach. J Hand Surg Am 2005;30:1145-52.

14. Inoue G, Shionoya K. Late treatment of unreduced perilunate dislocations. J Hand Surg Br 1999;24:221-5.

15. Goodman AD, Harris AP, Gil JA, Park J, Raducha J, Got CJ. Evaluation, Management, and Outcomes of Lunate and Perilunate Dislocations. Orthopedics 2019;42:e1-e6.

16. Apergis E, Maris J, Theodoratos G, Pavlakis D, Antoniou N. Perilunate dislocations and fracture-dislocations. Closed and early open reduction compared in 28 cases. Acta Orthop Scand Suppl 1997;275:55-9.
17. Forli A, Courvoisier A, Wimsey S, Corcella D, Moutet F. Perilunate dislocations and transscaphoid perilunate fracture-dislocations: a retrospective study with minimum ten-year follow-up. J Hand Surg Am 2010;35:62-8.

18. Trumble T, Verheyden J. Treatment of isolated perilunate and lunate dislocations with combined dorsal and volar approach and intraosseous cerclage wire. The Journal of Hand Surgery 2004;29:412-7.

19. Adkison JW, Chapman MW. Treatment of acute lunate and perilunate dislocations. Clin Orthop Relat Res 1982;164:199-207.

20. Najarian R, Nourbakhsh A, Capo J, Tan V. Perilunate injuries. Hand 2011;6:1-7.

21. Başar H, Başar B, Erol B, Tetik C. Isolated volar surgical approach for the treatment of perilunate and lunate dislocations. Indian J Orthop 2014:48:301-5. 